\title{
BOUNDARY VALUE PROBLEM WITH INTEGRAL CONDITIONS FOR A LINEAR THIRD-ORDER EQUATION
}

\section{DENCHE AND A. MEMOU}

Received 6 March 2003 and in revised form 29 July 2003

We prove the existence and uniqueness of a strong solution for a linear third-order equation with integral boundary conditions. The proof uses energy inequalities and the density of the range of the generated operator.

\section{Introduction}

In the rectangle $\Omega=[0,1] \times[0, T]$, we consider the equation

$$
£ u=\frac{\partial^{3} u}{\partial t^{3}}+\frac{\partial}{\partial x}\left(a(x, t) \frac{\partial u}{\partial x}\right)=f(x, t)
$$

with the initial conditions

$$
u(x, 0)=0, \quad \frac{\partial u}{\partial t}(x, 0)=0, \quad x \in(0,1)
$$

the final condition

$$
\frac{\partial^{2} u}{\partial t^{2}}(x, T)=0, \quad x \in(0,1)
$$

the Dirichlet condition

$$
u(0, t)=0, \quad \forall t \in(0, T),
$$


554 Boundary value problem with integral conditions

and the integral condition

$$
\int_{0}^{1} u(x, t) d x=0, \quad \forall t \in(0, T) .
$$

In addition, we assume that the function $a(x, t)$ is bounded with

$$
0<a_{0} \leq a(x, t) \leq a_{1},
$$

and has bounded partial derivatives such that

$$
\begin{gathered}
c_{k}^{\prime} \leq \frac{\partial^{k} a}{\partial t^{k}}(x, t) \leq c_{k}, \quad \forall x \in(0,1), t \in(0, T), k=\overline{1,3}, \text { with } c_{1}^{\prime} \geq 0, \\
\left|\frac{\partial a}{\partial x}(x, t)\right| \leq b_{1}, \quad \text { for }(x, t) \in \Omega .
\end{gathered}
$$

Various problems arising in heat conduction $[4,6,14,15]$, chemical engineering [9], underground water flow [13], thermoelasticity [21], and plasmaphysics [19] can be reduced to the nonlocal problems with integral boundary conditions. This type of boundary value problems has been investigated in $[1,2,3,5,6,7,9,14,15,16,20,23]$ for parabolic equations, in $[18,22]$ for hyperbolic equations, and in $[10,11,12]$ for mixed-type equations. The basic tool in $[4,10,11,12,16,23]$ is the energy inequality method which, of course, requires appropriate multipliers and functional spaces. In this paper, we extend this method to the study of a linear third-order partial differential equation. This type of problems is encountered in the study of thermal conductivity [17] and microscale heat transfer [8].

\section{Preliminaries}

In this paper, we prove the existence and uniqueness of a strong solution of problem (1.1). For this, we consider the solution of problem (1.1) as a solution of the operator equation $L u=\mathcal{F}$, where $L$ is the operator with domain of definition $D(L)$ consisting of functions $u \in E$ such that $\sqrt{1-x}\left(\partial^{k+1} u / \partial t^{k} \partial x\right)(x, t) \in L^{2}(\Omega), k=\overline{0,3}$ and $u$ satisfies conditions (1.1d) and (1.1e). The operator $L$ is considered from $E$ to $F$, where $E$ is the Banach space of the functions $u, u \in L^{2}(\Omega)$, with the finite norm

$$
\begin{aligned}
\|u\|_{E}^{2}= & \int_{\Omega} \frac{(1-x)^{2}}{2}\left\{\left|\frac{\partial^{3} u}{\partial t^{3}}\right|^{2}+\left|\frac{\partial^{2} u}{\partial x^{2}}\right|^{2}\right\} d x d t \\
& +\int_{\Omega}\left(\frac{(1-x)^{2}}{2}\left|\frac{\partial u}{\partial x}\right|^{2}+|u|^{2}\right) d x d t
\end{aligned}
$$


and $F$ is the Hilbert space of the functions $\mathcal{F}=(f, 0,0,0), f \in L^{2}(\Omega)$, with the finite norm

$$
\|\mathcal{F}\|_{F}^{2}=\int_{\Omega}(1-x)^{2}|f|^{2} d x d t
$$

Then we establish an energy inequality

$$
\|u\|_{E} \leq k\|L u\|_{F}, \quad \forall u \in D(L),
$$

and we show that the operator $L$ has the closure $\bar{L}$.

Definition 2.1. A solution of the operator equation $\bar{L} u=\mathcal{F}$ is called a strong solution of problem (1.1).

Inequality (2.3) can be extended to $u \in D(\bar{L})$, that is,

$$
\|u\|_{E} \leq k\|\bar{L} u\|_{F^{\prime}} \quad \forall u \in D(\bar{L}) .
$$

From this inequality, we obtain the uniqueness of a strong solution, if it exists, and the equality of the sets $R(\bar{L})$ and $\overline{R(L)}$. Thus, to prove the existence of a strong solution of problem (1.1) for any $₹ \in F$, it remains to prove that the set $R(L)$ is dense in $F$.

\section{An energy inequality and its applications}

THEOREM 3.1. For any function $u \in D(L)$, there exists the a priori estimate

$$
\|u\|_{E} \leq k\|L u\|_{F}
$$

where

$$
k^{2}=\frac{17 \exp (c t)\left[5+4\left(b_{1}\right)^{2} /\left(c_{3}^{\prime}-3 c c_{2}+3 c^{2} c_{1}^{\prime}-c^{3} a_{1}-b_{1}^{2}\right)\right]+1}{\min \left(1, a_{0}^{2}, c_{3}^{\prime}-3 c c_{2}+3 c^{2} c_{1}^{\prime}-c^{3} a_{1}-b_{1}^{2}\right)},
$$

with the constant $c$ satisfying

$$
\begin{gathered}
\sup _{(x, t) \in \Omega}\left(\frac{1}{a} \frac{\partial a}{\partial t}\right) \leq c<\inf _{(x, t) \in \Omega}\left(\frac{1}{a} \frac{\partial a}{\partial t}+1\right), \\
c_{3}^{\prime}-3 c c_{2}+3 c^{2} c_{1}^{\prime}-c^{3} a_{1}-\left(b_{1}\right)^{2}>0, \\
c_{2}-2 c c_{1}^{\prime}+c^{2} a_{1}-c_{1}^{\prime}+c a_{1}<0 .
\end{gathered}
$$


556 Boundary value problem with integral conditions

Proof. Let

$$
M u=(1-x)^{2} \frac{\partial^{3} u}{\partial t^{3}}+2(1-x) J_{x} \frac{\partial^{3} u}{\partial t^{3}}
$$

where

$$
J_{x} u=\int_{0}^{x} u(\zeta, t) d \zeta .
$$

We consider the quadratic form

$$
\Phi(u, u)=\operatorname{Re} \int_{\Omega} \exp (-c t) £ u \overline{M u} d x d t
$$

with the constant $c$ satisfying (3.3), obtained by multiplying (1.1a) by $\exp (-c t) \overline{M u}$, integrating over $\Omega$, and taking the real part. Substituting the expression of $M u$ in (3.6), we obtain

$$
\begin{aligned}
& \operatorname{Re} \int_{\Omega} \exp (-c t) £ u \overline{M u} d x d t \\
& =\operatorname{Re} \int_{\Omega} \exp (-c t)(1-x)^{2}\left|\frac{\partial^{3} u}{\partial t^{3}}\right|^{2} d x d t \\
& \quad+2 \operatorname{Re} \int_{\Omega} \exp (-c t)(1-x) \frac{\partial^{3} u}{\partial t^{3}} J_{x} \frac{\partial^{3} u}{\partial t^{3}} d x d t \\
& \quad+\operatorname{Re} \int_{\Omega} \exp (-c t) \frac{\partial}{\partial x}\left(a(x, t) \frac{\partial u}{\partial x}\right) \overline{M u} d x d t
\end{aligned}
$$

Integrating the last two terms on the right-hand side by parts with respect to $x$ in (3.7) and using the Dirichlet condition (1.1d), we obtain

$$
\begin{aligned}
2 \operatorname{Re} \int_{0}^{1}(1-x) \exp (-c t) \frac{\partial^{3} u}{\partial t^{3}} J_{x} \frac{\partial^{3} \bar{u}}{\partial t^{3}} d x=\int_{0}^{1} \exp (-c t)\left|J_{x} \frac{\partial^{3} u}{\partial t^{3}}\right|^{2} d x \\
\operatorname{Re} \int_{\Omega} \exp (-c t) \frac{\partial}{\partial x}\left(a \frac{\partial u}{\partial x}\right) \overline{M u} d x d t \\
=-\operatorname{Re} \int_{\Omega} \exp (-c t)(1-x)^{2} a \frac{\partial u}{\partial x} \frac{\partial^{4} \bar{u}}{\partial t^{3} \partial x} d x d t \\
-2 \operatorname{Re} \int_{\Omega} \exp (-c t) \frac{\partial a}{\partial x} u J_{x} \frac{\partial^{3} \bar{u}}{\partial t^{3}} d x d t \\
-2 \operatorname{Re} \int_{\Omega} \exp (-c t) a u \frac{\partial^{3} \bar{u}}{\partial t^{3}} d x d t
\end{aligned}
$$


Integrating each term by parts in (3.9) with respect to $t$ and using the initial and final conditions (1.1b) and (1.1c), we get

$$
\begin{aligned}
\operatorname{Re} \int_{\Omega} & \exp (-c t) \frac{\partial}{\partial x}\left(a \frac{\partial u}{\partial x}\right) \overline{M u} d x d t \\
= & -2 \operatorname{Re} \int_{\Omega} \exp (-c t) \frac{\partial a}{\partial x} u J_{x} \frac{\partial^{3} \bar{u}}{\partial t^{3}} d x d t \\
& +\int_{\Omega} \exp (-c t)\left(\frac{\partial^{3} a}{\partial t^{3}}-3 c \frac{\partial^{2} a}{\partial t^{2}}+3 c^{2} \frac{\partial a}{\partial t}-c^{3} a\right) \\
& \quad-\quad\left[\frac{(1-x)^{2}}{2}\left|\frac{\partial u}{\partial x}\right|^{2}+|u|^{2}\right] d x d t \\
& +\int_{\Omega}^{1} \exp (-c t)\left(\frac{\partial a}{\partial t}-c a\right)\left[\frac{(1-x)^{2}}{2}\left|\frac{\partial^{2} u}{\partial t \partial x}\right|^{2}+\left|\frac{\partial u}{\partial t}\right|^{2}\right] d x d t \\
& \left.-\left.\int_{0}^{1} \exp (-c t)\left(\frac{\partial^{2} a}{\partial t^{2}}-2 c \frac{\partial a}{\partial t}+c^{2} a\right)\left[\frac{(1-x)^{2}}{2}\left|\frac{\partial^{2} u}{\partial t \partial x}\right|^{2}+\left|\frac{\partial u}{\partial t}\right|^{2}\right] d x\right|_{T=t} ^{2}\left|\frac{\partial u}{\partial x}\right|^{2}+|u|^{2}\right]\left.d x\right|_{t=T} \\
& +\left.\operatorname{Re} \int_{0}^{1} \exp (-c t)\left(\frac{\partial a}{\partial t}-c a\right)\left\{(1-x)^{2} \frac{\partial^{2} \bar{u}}{\partial t \partial x} \frac{\partial u}{\partial x}+2 u \frac{\partial \bar{u}}{\partial t}\right\}\right|_{T=t} d x
\end{aligned}
$$

Substituting (3.8) and (3.10) in (3.7) and using conditions (1.2), (1.3), and (3.3), we obtain

$$
\begin{aligned}
& \int_{\Omega} \exp (-c t)(1-x)^{2}\left|\frac{\partial^{3} u}{\partial t^{3}}\right|^{2} d x d t \\
& \quad+\int_{\Omega} \exp (-c t)\left\{c_{3}^{\prime}-3 c c_{2}+3 c^{2} c_{1}^{\prime}-c^{3} a_{1}-b_{1}^{2}\right\} \\
& \quad \times\left[\frac{(1-x)^{2}}{2}\left|\frac{\partial u}{\partial x}\right|^{2}+|u|^{2}\right] d x d t \\
& \leq \operatorname{Re} \int_{\Omega} \exp (-c t) £ u M \bar{u} d x d t
\end{aligned}
$$

Again, substituting the expression of $M u$ in (3.11) and using elementary inequality, we get 
558 Boundary value problem with integral conditions

$$
\begin{aligned}
& \int_{\Omega} \exp (-c t) \frac{(1-x)^{2}}{2}\left|\frac{\partial^{3} u}{\partial t^{3}}\right|^{2} d x d t \\
& \quad+\int_{\Omega} \exp (-c t)\left\{c_{3}^{\prime}-3 c c_{2}+3 c^{2} c_{1}^{\prime}-c^{3} a_{1}-b_{1}^{2}\right\} \\
& \quad \times\left[\frac{(1-x)^{2}}{2}\left|\frac{\partial u}{\partial x}\right|^{2}+|u|^{2}\right] d x d t \\
& \leq 17 \int_{\Omega} \exp (-c t)(1-x)^{2}|f|^{2} d x d t .
\end{aligned}
$$

By virtue of (1.1a), we have

$$
\begin{aligned}
\int_{\Omega} a_{0} \mid & \left.\frac{\partial^{2} u}{\partial x^{2}}\right|^{2} \frac{(1-x)^{2}}{2} d x d t \\
\leq & \int_{\Omega}(1-x)^{2}|f|^{2} d x d t+\int_{\Omega} 2(1-x)^{2}\left|\frac{\partial^{3} u}{\partial t^{3}}\right|^{2} d x d t \\
& +4 \int_{\Omega} b_{1}^{2}\left\{\frac{(1-x)^{2}}{2}\left|\frac{\partial u}{\partial x}\right|^{2}+|u|^{2}\right\} d x d t .
\end{aligned}
$$

This last inequality combined with (3.12) yields

$$
\begin{aligned}
& \int_{\Omega} \frac{(1-x)^{2}}{2}\left|\frac{\partial^{3} u}{\partial t^{3}}\right|^{2} d x d t \\
& \quad+\int_{\Omega}\left(c_{3}^{\prime}-3 c c_{2}+3 c^{2} c_{1}^{\prime}-c^{3} a_{1}-b_{1}^{2}\right)\left\{\frac{(1-x)^{2}}{2}\left|\frac{\partial u}{\partial x}\right|^{2}+|u|^{2}\right\} d x d t \\
& \quad+\int_{\Omega} a_{0}^{2} \frac{(1-x)^{2}}{2}\left|\frac{\partial^{2} u}{\partial x^{2}}\right|^{2} d x d t \\
& \leq\left\{17 \exp (c T)\left[5+\frac{4 b_{1}^{2}}{c_{3}^{\prime}-3 c c_{2}+3 c^{2} c_{1}^{\prime}-c^{3} a_{1}-b_{1}^{2}}\right]+1\right\} \\
& \times \int_{\Omega}(1-x)^{2}|f|^{2} d x d t .
\end{aligned}
$$

Thus, this inequality implies

$$
\begin{gathered}
\int_{\Omega} \frac{(1-x)^{2}}{2}\left\{\left|\frac{\partial^{3} u}{\partial t^{3}}\right|^{2}+\left|\frac{\partial^{2} u}{\partial x^{2}}\right|^{2}\right\} d x d t+\int_{\Omega} \frac{(1-x)^{2}}{2}\left|\frac{\partial u}{\partial x}\right|^{2}+|u|^{2} d x d t \\
\leq k^{2} \int_{\Omega}(1-x)^{2}|f|^{2} d x d t
\end{gathered}
$$


where

$$
k^{2}=\frac{17 \exp (c T)\left[5+4 b_{1}^{2} /\left(c_{3}^{\prime}-3 c c_{2}+3 c^{2} c_{1}^{\prime}-c^{3} a_{1}-b_{1}^{2}\right)\right]+1}{\min \left(1, a_{0}^{2}, c_{3}^{\prime}-3 c c_{2}+3 c^{2} c_{1}^{\prime}-c^{3} a_{1}-b_{1}^{2}\right)} .
$$

Then,

$$
\|u\|_{E} \leq k\|L u\|_{F}, \quad \forall u \in D(L)
$$

Thus, we obtain the desired inequality.

LEMMA 3.2. The operator L from $E$ to $F$ admits a closure.

Proof. Suppose that $\left(u_{n}\right) \in D(L)$ is a sequence such that

$$
u_{n} \longrightarrow 0 \quad \text { in } E, \quad L u_{n} \longrightarrow \mathcal{F} \quad \text { in } F \text {. }
$$

We need to show that $₹=0$. We introduce the operator

$$
£_{0} v=-(1-x)^{2} \frac{\partial^{3} v}{\partial t^{3}}+\frac{\partial}{\partial x}\left\{a(x, t) \frac{\partial}{\partial x}\left[(1-x)^{2} v\right]\right\}
$$

with domain $D\left(£_{0}\right)$ consisting of functions $v \in W_{2}^{2,3}(\Omega)$ satisfying

$$
\left.v\right|_{t=0}=0,\left.\quad \frac{\partial v}{\partial t}\right|_{t=0}=0,\left.\quad \frac{\partial^{2} v}{\partial t^{2}}\right|_{t=0}=0,\left.\quad v\right|_{x=0}=0,\left.\quad \frac{\partial v}{\partial x}\right|_{x=0}=0 .
$$

We note that $D\left(£_{0}\right)$ is dense in the Hilbert space obtained by completing $L^{2}(\Omega)$ with respect to the norm

$$
\int_{\Omega}(1-x)^{2}|v|^{2} d x d t=\|v\|^{2}
$$

Since

$$
\begin{aligned}
\int_{\Omega}(1-x)^{2} f \bar{v} d x d t & =\lim _{n \rightarrow+\infty} \int_{\Omega}(1-x)^{2} £ u_{n} \bar{v} d x d t \\
& =\lim _{n \rightarrow+\infty} \int_{\Omega} u_{n} £_{0} \bar{v} d x d t=0
\end{aligned}
$$

for any function $v \in D\left(£_{0}\right)$, it follows that $f=0$. 
560 Boundary value problem with integral conditions

Theorem 3.1 is valid for a strong solution, then we have the inequality

$$
\|u\|_{E} \leq k\|\bar{L} u\|_{F^{\prime}} \quad \forall u \in D(\bar{L})
$$

Hence we obtain the following corollary.

Corollary 3.3. A strong solution of problem (1.1) is unique if it exists, and depends continuously on $\mathcal{F}$.

Corollary 3.4. The range $R(\bar{L})$ of the operator $\bar{L}$ is closed in $F$, and $R(\bar{L})=$ $\overline{R(L)}$.

\section{Solvability of problem (1.1)}

To prove the solvability of problem (1.1), it is sufficient to show that $R(L)$ is dense in $F$. The proof is based on the following lemma.

LEMMA 4.1. Suppose that $a(x, t)$ and its derivatives $\partial^{4} a / \partial t^{3} \partial x$ and $\partial^{2} a / \partial t \partial x$ are bounded. Let $D_{0}(L)=\left\{u \in D(L): u(x, 0)=0,(\partial u / \partial t)(x, 0)=0,\left(\partial^{2} u /\right.\right.$ $\left.\left.\partial t^{2}\right)(x, T)=0\right\}$. If, for $u \in D_{0}(L)$ and for some functions $w \in L^{2}(\Omega)$,

$$
\int_{\Omega}(1-x) £ u \bar{w} d x d t=0
$$

then $w=0$.

Proof. Equality (4.1) can be written as follows:

$$
\int_{\Omega}(1-x) \bar{w} \frac{\partial^{3} u}{\partial t^{3}} d x d t=-\int_{\Omega} \frac{\partial}{\partial x}\left(a(1-x) \frac{\partial u}{\partial x}\right)\left\{\bar{w}-\int_{0}^{x} \frac{\bar{w}}{1-\zeta} d \zeta\right\} d x d t
$$

For a given $w(x, t)$, we introduce the function $v(x, t)$ such that

$$
v(x, t)=w(x, t)-\int_{0}^{x} \frac{w(\zeta, t)}{1-\zeta} d \zeta
$$

From (4.3), we conclude that $\int_{0}^{1} v(x, t) d x=0$, and thus, we have

$$
\int_{\Omega} \frac{\partial^{3} u}{\partial t^{3}} \overline{N v} d x d t=-\int_{\Omega} A(t) u \bar{v} d x d t
$$

where $A(t) u=(\partial / \partial x)(a(1-x)(\partial u / \partial x))$ and $N v=(1-x) v+J v$. 
Following [23], we introduce the smoothing operators

$$
J_{\mathcal{\varepsilon}}^{-1}=\left(I-\epsilon\left(\frac{\partial^{3}}{\partial t^{3}}\right)\right)^{-1}, \quad\left(J_{\mathcal{\varepsilon}}^{-1}\right)^{*}=\left(I+\epsilon\left(\frac{\partial^{3}}{\partial t^{3}}\right)\right)^{-1},
$$

with respect to $t$, which provide the solutions of the respective problems

$$
\begin{array}{llll}
g_{\epsilon}-\epsilon \frac{\partial^{3} g_{\varepsilon}}{\partial t^{3}}=g, & g_{\epsilon}(0)=0, & \frac{\partial g_{\epsilon}}{\partial t}(0)=0, & \frac{\partial^{2} g_{\epsilon}}{\partial t^{2}}(T)=0, \\
g_{\epsilon}^{*}+\epsilon \frac{\partial^{3} g_{\epsilon}^{*}}{\partial t^{3}}=g^{3}, & g_{\epsilon}^{*}(0)=0, & \frac{\partial g_{\epsilon}^{*}}{\partial t}(T)=0, & \frac{\partial^{2} g_{\epsilon}^{*}}{\partial t^{2}}(T)=0 .
\end{array}
$$

We also have the following properties: for any $g \in L^{2}(0, T)$, the functions $J_{\epsilon}^{-1}(g),\left(J_{\epsilon}^{-1}\right)^{*} g \in W_{2}^{3}(0, T)$. If $g \in D(L)$, then $J_{\epsilon}^{-1}(g) \in D(L)$ and we have

$$
\begin{array}{cc}
\lim \left\|\left(J_{\epsilon}^{-1}\right)^{*} g-g\right\|_{L^{2}[0, T]}=0 & \text { for } \epsilon \longrightarrow 0, \\
\lim \left\|\left(J_{\epsilon}^{-1}\right) g-g\right\|_{L^{2}[0, T]}=0 & \text { for } \epsilon \longrightarrow 0 .
\end{array}
$$

Substituting the function $u$ in (4.4) by the smoothing function $u_{\varepsilon}$ and using the relation

$$
A(t) u_{\varepsilon}=J_{\varepsilon}^{-1} A u-\epsilon J_{\varepsilon}^{-1} \beta_{\epsilon}(t) u_{\varepsilon}
$$

where

$$
\beta_{\epsilon}(t) u_{\varepsilon}=3 \frac{\partial^{2} A(t)}{\partial t^{2}} \frac{\partial u_{\varepsilon}}{\partial t}+3 \frac{\partial A(t)}{\partial t} \frac{\partial^{2} u_{\varepsilon}}{\partial^{2} t}+\frac{\partial^{3} A(t)}{\partial t^{3}} u_{\varepsilon}
$$

we obtain

$$
-\int_{\Omega} u N \frac{\partial^{3}{\overline{v_{\varepsilon}}}^{*}}{\partial^{3} t} d x d t=\int_{\Omega} A(t) u \overline{v_{\varepsilon}^{*}} d x d t-\epsilon \int_{\Omega} \beta_{\epsilon}(t) u_{\varepsilon} \overline{v_{\varepsilon}^{*}} d x d t
$$

Passing to the limit, the equality in the relation (4.10) remains true for all functions $u \in L^{2}(\Omega)$ such that $(1-x)(\partial u / \partial x),(\partial / \partial x)((1-x)(\partial u / \partial x)) \in$ $L^{2}(\Omega)$, and satisfying condition $(1.1 \mathrm{~d})$. 
562 Boundary value problem with integral conditions

The operator $A(t)$ has a continuous inverse in $L^{2}(0,1)$ defined by

$$
\begin{aligned}
A^{-1}(t) g= & -\int_{0}^{x} \frac{1}{1-\zeta} \frac{1}{a(\zeta, t)} \int_{0}^{\zeta} g(\eta, t) d \eta d \zeta \\
& +C(t) \int_{0}^{x} \frac{1}{1-\zeta} \frac{1}{a(\zeta, t)} d \zeta,
\end{aligned}
$$

where

$$
C(t)=\frac{\int_{0}^{1}(d \zeta / a(\zeta, t)) \int_{0}^{\zeta} g(\eta, t) d \eta}{\int_{0}^{1}(d \zeta / a(\zeta, t))} .
$$

Then, we have $\int_{0}^{1} A^{-1}(t) g d x=0$, hence the function $u_{\varepsilon}=\left(J_{\varepsilon}\right)^{-1} u$ can be represented in the form

$$
u_{\varepsilon}=\left(J_{\varepsilon}\right)^{-1} A^{-1}(t) A(t) u
$$

Then

$$
\begin{aligned}
B_{\varepsilon}(t) g= & \frac{\partial^{4} a}{\partial t^{3} \partial x} J_{\varepsilon}^{-1}\left[\frac{1}{a(x, t)}\left(\int_{0}^{x} g(\eta, t) d \eta-C(t)\right)\right] \\
& +\frac{\partial^{3} a}{\partial t^{3}} J_{\varepsilon}^{-1}\left[\frac{g}{a}-\frac{a_{x}}{a^{2}(x, t)}\left(\int_{0}^{x} g(\eta, t) d \eta-C(t)\right)\right] \\
& +3 \frac{\partial}{\partial t} \frac{\partial^{2} a}{\partial t^{2} \partial x} \frac{\partial}{\partial t} J_{\varepsilon}^{-1} \frac{1}{a(x, t)}\left(\int_{0}^{x} g(\eta, t) d \eta-C(t)\right) \\
& +\frac{\partial a}{\partial t} \frac{\partial}{\partial t} J_{\varepsilon}^{-1} \frac{g}{a}-\frac{a_{x}}{a^{2}(x, t)}\left(\int_{0}^{x} g(\eta, t) d \eta-C(t)\right) .
\end{aligned}
$$

The adjoint of $B_{\varepsilon}(t)$ has the form

$$
\begin{aligned}
B_{\varepsilon}^{*}(t)= & \frac{1}{a}\left(J_{\varepsilon}^{-1}\right)^{*}\left[\frac{\partial^{3} a}{\partial t^{3}} \bar{h}\right]+\frac{3}{a}\left(J_{\varepsilon}^{-1}\right)^{*} \frac{\partial}{\partial t}\left(\frac{\partial a}{\partial t} \frac{\partial \bar{h}}{\partial t}\right) \\
& +\left(G_{\varepsilon} h\right)(x)-\frac{\int_{0}^{x}(1 / a(\eta, t)) d \eta}{\int_{0}^{1}(1 / a(x, t)) d x}\left(G_{\varepsilon} h\right)(1),
\end{aligned}
$$


where

$$
\begin{aligned}
\left(G_{\epsilon} h\right)(x)= & \int_{0}^{x}\left(-\frac{3}{a(\zeta, t)}\left(J_{\epsilon}^{-1}\right)^{*} \frac{\partial}{\partial t}\left(\frac{\partial^{2}}{\partial t \partial \zeta} \frac{\partial h}{\partial t}\right)\right. \\
& +3 \frac{\partial a}{\partial \zeta} \frac{1}{a^{2}(\zeta, t)}\left(J_{\epsilon}^{-1}\right)^{*} \frac{\partial}{\partial t}\left(\frac{\partial a}{\partial t} \frac{\partial h}{\partial t}\right) \\
& \left.-\frac{1}{a(\zeta, t)}\left(J_{\varepsilon}^{-1}\right)^{*}\left(\frac{\partial^{4} a}{\partial t^{3} \partial \zeta} h\right)+\frac{\partial a}{\partial \zeta} \frac{1}{a^{2}(\zeta, t)}\left(J_{\varepsilon}^{-1}\right)^{*}\left(\frac{\partial^{3} a}{\partial t^{3}} h\right)\right) d \zeta .
\end{aligned}
$$

Consequently, equality (4.10) becomes

$$
-\int_{\Omega} u N \frac{\partial^{3} \overline{v_{\varepsilon}^{*}}}{\partial t^{3}} d x d t=\int_{\Omega} A(t) u \overline{h_{\varepsilon}} d x d t
$$

where $h_{\varepsilon}=v_{\varepsilon}^{*}-\varepsilon B_{\varepsilon}^{*} v_{\varepsilon}^{*}$.

The left-hand side of (4.17) is a continuous linear functional of $u$. Hence the function $h_{\varepsilon}$ has the derivatives $(1-x)\left(\partial h_{\varepsilon} / \partial x\right),(\partial / \partial x)((1-$ $\left.x)\left(\partial h_{\varepsilon} / \partial x\right)\right) \in L^{2}(\Omega)$ and the following conditions are satisfied: $\left.h_{\varepsilon}\right|_{x=0}=$ $0,\left.h_{\varepsilon}\right|_{x=1}=0$, and $\left.(1-x)\left(\partial h_{\varepsilon} / \partial x\right)\right|_{x=1}=0$.

From the equality

$$
\begin{aligned}
(1-x) \frac{\partial h_{\varepsilon}}{\partial x}= & {\left[I-\varepsilon \frac{1}{a}\left(J_{\varepsilon}^{-1}\right)^{*} \frac{\partial^{3} a}{\partial t^{3}}\right](1-x) \frac{\partial v_{\varepsilon}^{*}}{\partial x} } \\
& -3 \varepsilon \frac{1}{a}\left(J_{\varepsilon}^{-1}\right)^{*} \frac{\partial}{\partial t}\left(\frac{\partial a}{\partial t} \frac{\partial}{\partial t}(1-x) \frac{\partial v_{\varepsilon}^{*}}{\partial x}\right)
\end{aligned}
$$

and since the operator $\left(J_{\varepsilon}^{-1}\right)^{*}$ is bounded in $L^{2}(\Omega)$, for sufficiently small $\varepsilon$, we have $\left\|\varepsilon(1 / a)\left(J_{\varepsilon}^{-1}\right)^{*}\left(\partial^{3} a / \partial t^{3}\right)\right\|<1$. Hence the operator $I-\varepsilon(1 / a)\left(J_{\varepsilon}^{-1}\right)^{*}$ $\left(\partial^{3} a / \partial t^{3}\right)$ has a bounded inverse in $L^{2}(\Omega)$. We conclude that $(1-x)\left(\partial v_{\varepsilon}^{*} /\right.$ $\partial x) \in L^{2}(\Omega)$.

Similarly, we conclude that $(\partial / \partial x)\left((1-x)\left(\partial v_{\varepsilon}^{*} / \partial x\right)\right)$ exists and belongs to $L^{2}(\Omega)$, and the following conditions are satisfied:

$$
\left.v_{\varepsilon}^{*}\right|_{x=0}=0,\left.\quad v_{\varepsilon}^{*}\right|_{x=1}=0,\left.\quad(1-x) \frac{\partial v_{\varepsilon}^{*}}{\partial x}\right|_{x=1}=0 .
$$

Substituting $u=\int_{0}^{t} \int_{0}^{\eta} \int_{\zeta}^{T} \exp (c \tau) v_{\varepsilon}^{*}(\tau) d \tau d \zeta d \eta$ in (4.4), where the constant $c$ satisfies (3.3), we obtain

$$
\int_{\Omega} \exp (c t) v_{\mathcal{\varepsilon}}^{*} N \bar{v} d x d t=-\int_{\Omega} A(t) u \bar{v} d x d t .
$$


564 Boundary value problem with integral conditions

Using the properties of smoothing operators, we have

$$
\int_{\Omega} \exp (c t) v_{\varepsilon}^{*} N \bar{v} d x d t=-\int_{\Omega} A(t) u \overline{v_{\varepsilon}^{*}} d x d t-\varepsilon \int_{\Omega} A(t) u \frac{\partial^{3} \overline{v_{\varepsilon}^{*}}}{\partial t^{3}} d x d t
$$

and from

$$
\begin{aligned}
\varepsilon \operatorname{Re} \int_{\Omega} A(t) u \frac{\partial^{3} \overline{v_{\varepsilon}^{*}}}{\partial t^{3}} d x d t= & \varepsilon \int_{\Omega}(1-x) a \frac{\partial u}{\partial x} \frac{\partial}{\partial x} \frac{\partial^{3} \overline{v_{\varepsilon}^{*}}}{\partial t^{3}} d x d t \\
= & -\varepsilon \operatorname{Re} \int_{\Omega}(1-x) \frac{\partial a}{\partial t} \frac{\partial u}{\partial x} \frac{\partial^{2}}{\partial t^{2}} \frac{\partial \overline{v_{\varepsilon}^{*}}}{\partial x} d x d t \\
& +\varepsilon \operatorname{Re} \int_{\Omega}(1-x) \frac{\partial a}{\partial t} \frac{\partial^{2} u}{\partial t \partial x} \frac{\partial}{\partial t} \frac{\partial \overline{v_{\varepsilon}^{*}}}{\partial x} d x d t \\
& +\varepsilon \int_{\Omega} a \exp (-c t)(1-x)\left|\frac{\partial \overline{v_{\varepsilon}^{*}}}{\partial x}\right|^{2} d x d t \\
& +\varepsilon \operatorname{Re} \int_{\Omega}(1-x) \frac{\partial a}{\partial t} \frac{\partial^{2} u}{\partial t \partial x} \frac{\partial \overline{v_{\varepsilon}^{*}}}{\partial x} d x d t
\end{aligned}
$$

we have

$$
\begin{aligned}
\varepsilon \operatorname{Re} \int_{\Omega} & A(t) u \frac{\partial^{3} \overline{v_{\varepsilon}^{*}}}{\partial t^{3}} d x d t \\
\geq & \varepsilon \int_{\Omega} a \exp (+c t)(1-x)\left|\frac{\partial \overline{v_{\varepsilon}^{*}}}{\partial x}\right|^{2} d x d t \\
& -\varepsilon \int_{\Omega}(1-x) \frac{1}{4 a}\left(\frac{\partial a}{\partial t}\right)^{2} \exp (-c t)\left|\frac{\partial^{3} u}{\partial t^{2} \partial x}\right|^{2} d x d t \\
& -\varepsilon \int_{\Omega} a \exp (+c t)(1-x)\left|\frac{\partial v_{\varepsilon}^{*}}{\partial x}\right|^{2} d x d t \\
& -\varepsilon \int_{\Omega} \frac{1-x}{2}\left(\frac{\partial a}{\partial t}\right)^{2} \exp (-c t)\left|\frac{\partial u}{\partial x}\right|^{2} d x d t \\
& -\varepsilon \int_{\Omega} \exp (+c t) \frac{1-x}{2}\left|\frac{\partial^{3} \overline{v_{\varepsilon}^{*}}}{\partial t^{2} \partial x}\right|^{2} d x d t \\
& -\varepsilon \int_{\Omega} \exp (+c t) \frac{1}{2}\left|\frac{\partial^{2} \overline{v_{\varepsilon}^{*}}}{\partial t \partial x}\right|^{2} d x d t \\
& -\varepsilon \int_{\Omega} \frac{1-x}{2}\left(\frac{\partial a}{\partial t}\right)^{2} \exp (-c t)\left|\frac{\partial^{2} u}{\partial x \partial t}\right|^{2} d x d t
\end{aligned}
$$


Integrating the first term on the right-hand side by parts in (4.21), we obtain

$$
\begin{aligned}
& \operatorname{Re} \int_{\Omega} A(t) u v_{\varepsilon}^{*} d x d t \\
& \geq-\frac{3}{2} \int_{\Omega}(1-x) \exp (-c t)\left(\frac{\partial a}{\partial t}-c a\right)\left|\frac{\partial^{2} \bar{u}}{\partial t \partial x}\right|^{2} d x d t \\
&+\left.\frac{1}{2} \int_{0}^{1}(1-x) \exp (-c t)\left(a-\left|\frac{\partial a}{\partial t}-c a\right|\right)\left|\frac{\partial^{2} \bar{u}}{\partial t \partial x}\right|^{2} d x\right|_{t=T} \\
&-\left.\frac{1}{2} \int_{0}^{1}(1-x) \exp (-c t)\left\{\frac{\partial^{2} a}{\partial t^{2}}-2 c \frac{\partial a}{\partial t}+c^{2} a+\left|\frac{\partial a}{\partial t}-c a\right|\right\}\left|\frac{\partial u}{\partial x}\right|^{2}\right|_{t=T} d x \\
&+\frac{1}{2} \int_{\Omega}(1-x) \exp (-c t)\left\{\frac{\partial^{3} a}{\partial t^{3}}-3 c \frac{\partial^{2} a}{\partial t^{2}}+3 c^{2} \frac{\partial a}{\partial t}-c^{3} a\right\}\left|\frac{\partial u}{\partial x}\right|^{2} d x d t .
\end{aligned}
$$

Combining (4.23) and (4.24), we get

$$
\begin{aligned}
\operatorname{Re} \int_{\Omega} & \exp (c t) v_{\varepsilon}^{*} N \bar{v} d x d t \\
\leq & \frac{3}{2} \int_{\Omega}(1-x) \exp (-c t)\left(c_{1}-c a_{0}\right)\left|\frac{\partial^{2} \bar{u}}{\partial t \partial x}\right|^{2} d x d t \\
& -\left.\frac{1}{2} \int_{0}^{1}(1-x) \exp (-c t)\left\{a_{0}-c_{1}^{\prime}-c a_{1}\right\}\left|\frac{\partial^{2} u}{\partial t \partial x}\right|^{2} d x\right|_{t=T}\left|\frac{\partial u}{2}\right|_{t=T} d x \\
& +\frac{1}{2} \int_{0}^{1}(1-x) \exp (-c t)\left\{c_{2}-2 c_{1}^{\prime} c-c^{2} a_{1}-c_{1}^{\prime}+c a_{1}\right\}\left|\frac{\partial u}{\partial x}\right|^{2} d x d t \\
& -\left.\frac{1}{2} \int_{\Omega}(1-x) \exp (-c t)\left\{c_{3}^{\prime}-3 c_{2} c+3 c^{2} c_{1}^{\prime}-c^{3} a_{1}\right\}\left|\frac{c_{1}^{2}}{\partial x}\right| \frac{\partial^{3} \bar{u}}{\partial t^{2} \partial x}\right|^{2} d x d t \\
& +\varepsilon \int_{\Omega}(1-x) \exp (-c t) \frac{c_{1}^{2}}{4 a_{0}}\left|\frac{\partial u}{\partial x}\right|^{2} d x d t \\
& +\left.\int_{\Omega}(1-x) \exp (-c t) \frac{\partial^{3} v_{\varepsilon}^{*}}{\partial t^{2} \partial x}\right|^{2} d x d t \\
& +\int_{\Omega} \frac{1-x}{2} \exp (c t) \\
& +\int_{\Omega}(1-x) \exp (-c t) \frac{c_{1}^{2}}{2}\left|\frac{\partial^{2} \bar{u}}{\partial t \partial x}\right|^{2} d x d t \\
& \left.+\int_{\Omega} \frac{1-x}{2} \exp (c t)\left|\frac{\partial^{2} v_{\varepsilon}^{*}}{\partial t \partial x}\right|^{2} d x d t\right) .
\end{aligned}
$$


566 Boundary value problem with integral conditions

Using conditions (3.3) and inequalities (4.23) and (4.24), we obtain

$$
\operatorname{Re} \int_{\Omega} \exp (c t) v N \bar{v} d x d t \leq 0, \quad \text { as } \varepsilon \longrightarrow 0
$$

Since $\operatorname{Re} \int_{\Omega} \exp (c t) v J_{x} \bar{v} d x d t=0$, then $v=0$ a.e.

Finally, from the equality $(1-x) v+J_{x} v=(1-x) w$, we conclude $w=0$.

THEOREM 4.2. The range $R(\bar{L})$ of $\bar{L}$ coincides with $F$.

Proof. Since $F$ is Hilbert space, then $R(\bar{L})=F$ if and only if the relation

$$
\int_{\Omega}(1-x)^{2} £ u \bar{f} d x d t=0
$$

for arbitrary $u \in D_{0}(L)$ and $\mp \in F$, implies that $f=0$.

Taking $u \in D_{0}(L)$ in (4.27) and using Lemma 4.1, we obtain that $w=$ $(1-x) f=0$, then $f=0$.

\section{References}

[1] G. W. Batten Jr., Second-order correct boundary conditions for the numerical solution of the mixed boundary problem for parabolic equations, Math. Comp. 17 (1963), 405-413.

[2] S. A. Beilin, Existence of solutions for one-dimensional wave equations with nonlocal conditions, Electron. J. Differential Equations 2001 (2001), no. 76, 1-8.

[3] N. E. Benouar and N. I. Yurchuk, Mixed problem with an integral condition for parabolic equations with the Bessel operator, Differ. Equ. 27 (1991), no. 12, 1482-1487.

[4] A. Bouziani and N. E. Benouar, Mixed problem with integral conditions for a third order parabolic equation, Kobe J. Math. 15 (1998), no. 1, 47-58.

[5] B. Cahlon, D. M. Kulkarni, and P. Shi, Stepwise stability for the heat equation with a nonlocal constraint, SIAM J. Numer. Anal. 32 (1995), no. 2, 571-593.

[6] J. R. Cannon, The solution of the heat equation subject to the specification of energy, Quart. Appl. Math. 21 (1963), 155-160.

[7] , The One-Dimensional Heat Equation, Encyclopedia of Mathematics and Its Applications, vol. 23, Addison-Wesley Publishing, Massachusetts, 1984.

[8] A. U. Çoskun, Investigation of microscale heat transfer effects in nanoscale electronic devices by spectral methods, Abstracts of the Intenational Conference on Mathematical Modeling and Scientific Computing (Konya, Turkey), 2001.

[9] Y.S. Choi and K. Y. Chan, A parabolic equation with nonlocal boundary conditions arising from electrochemistry, Nonlinear Anal. 18 (1992), no. 4, 317-331. 
[10] M. Denche and A. L. Marhoune, High-order mixed-type differential equations with weighted integral boundary conditions, Electron. J. Differential Equations 2000 (2000), no. 60, 1-10.

[11] _ A three-point boundary value problem with an integral condition for parabolic equations with the Bessel operator, Appl. Math. Lett. 13 (2000), no. 6, 85-89.

[12] _ Mixed problem with nonlocal boundary conditions for a third-order partial differential equation of mixed type, Int. J. Math. Math. Sci. 26 (2001), no. 7, 417-426.

[13] R. E. Ewing and T. Lin, A class of parameter estimation techniques for fluid flow in porous media, Adv. in Water Res. 14 (1991), no. 2, 89-97.

[14] N. I. Ionkin, The solution of a certain boundary value problem of the theory of heat conduction with a nonclassical boundary condition, Differ. Uravn. 13 (1977), no. 2, 294-304 (Russian).

[15] N. I. Kamynin, A boundary value problem in the theory of heat conduction with non-classical boundary conditions, U.S.S.R. Comput. Math. and Math. Phys. 4 (1964), 33-59.

[16] A. V. Kartynnik, A three-point mixed problem with an integral condition with respect to the space variable for second-order parabolic equations, Differ. Equ. 26 (1990), no. 9, 1160-1166.

[17] V. I. Korzyuk and V. B. Kiselev, A boundary value problem for a third-order linear nonclassical equation generated by the heat equation, Vestsī Nats. Akad. Navuk Belarusī Ser. Fìz.-Mat. Navuk 2001 (2001), no. 3, 5-11 (Russian).

[18] L. S. Pulkina, A non-local problem with integral conditions for hyperbolic equations, Electron. J. Differential Equations 1999 (1999), no. 45, 1-6.

[19] A. A. Samarski, Some problems in the modern theory of differential equations, Differ. Uravn. 16 (1980), 1925-1935 (Russian).

[20] P. Shi, Weak solution to an evolution problem with a nonlocal constraint, SIAM J. Math. Anal. 24 (1993), no. 1, 46-58.

[21] P. Shi and M. Shillor, On design of contact patterns in one-dimensional thermoelasticity, Theoretical Aspects of Industrial Design (Wright-Patterson Air Force Base, Ohio, 1990), SIAM, Pennsylvania, 1992, pp. 76-82.

[22] V. F. Volkodavov and V. E. Zhukov, Two problems for the string vibration equation with integral conditions and special matching conditions on the characteristic, Differ. Equ. 34 (1998), no. 4, 501-505.

[23] N. I. Yurchuk, Mixed problem with an integral condition for certain parabolic equations, Differ. Equ. 22 (1986), 1457-1463.

M. Denche: Laboratoire Equations Différentielles, Département de Mathématiques, Faculté des Sciences, Université Mentouri, 25000 Constantine, Algeria

E-mail address: denech@wissal.dz

A. Memou: Laboratoire Equations Différentielles, Département de Mathématiques, Faculté des Sciences, Université Mentouri, 25000 Constantine, Algeria 


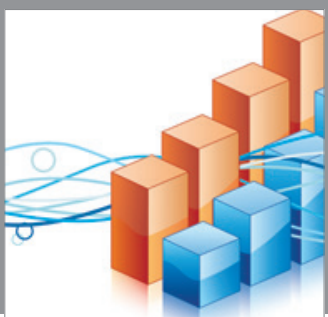

Advances in

Operations Research

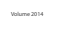

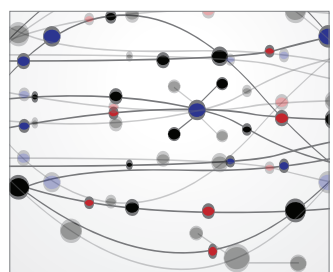

\section{The Scientific} World Journal
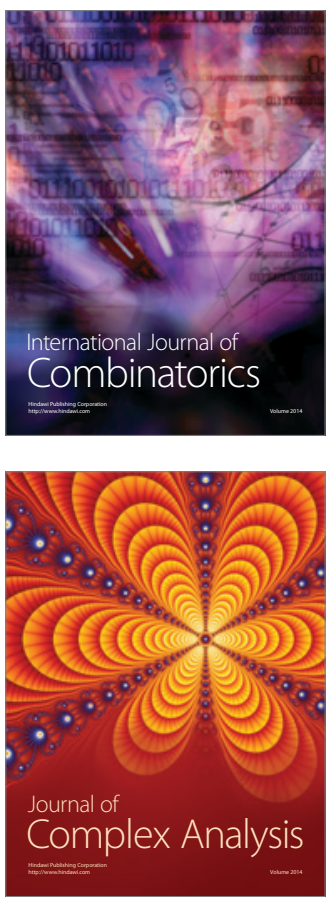

International Journal of

Mathematics and

Mathematical

Sciences
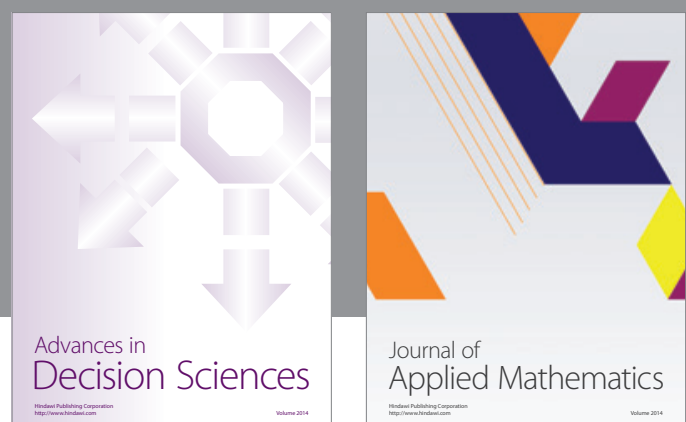

Journal of

Applied Mathematics
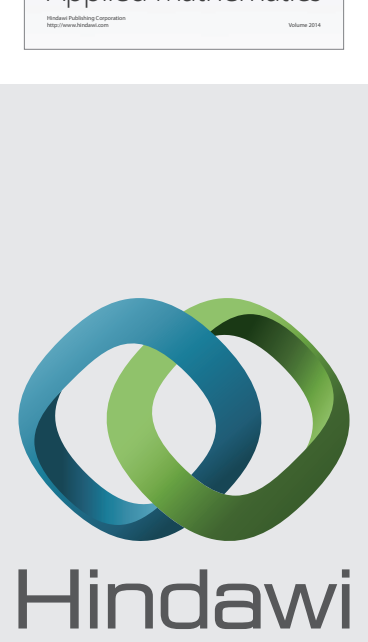

Submit your manuscripts at http://www.hindawi.com
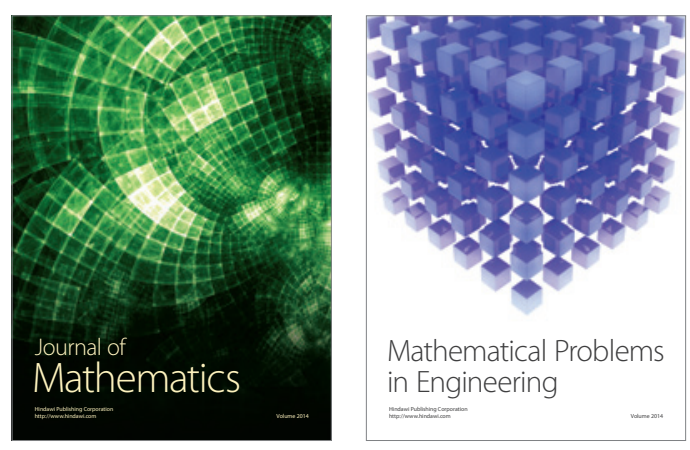

Mathematical Problems in Engineering
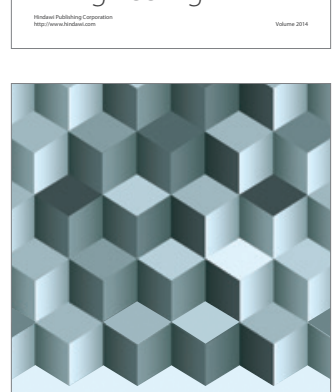

Journal of

Function Spaces
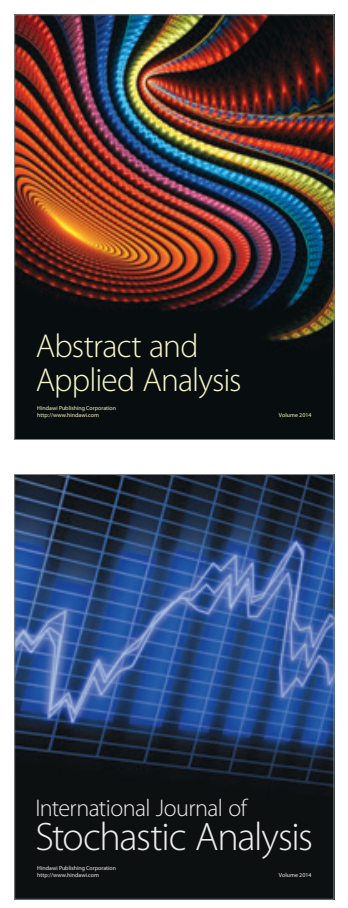

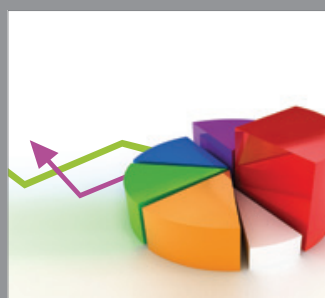

ournal of

Probability and Statistics

Promensencen
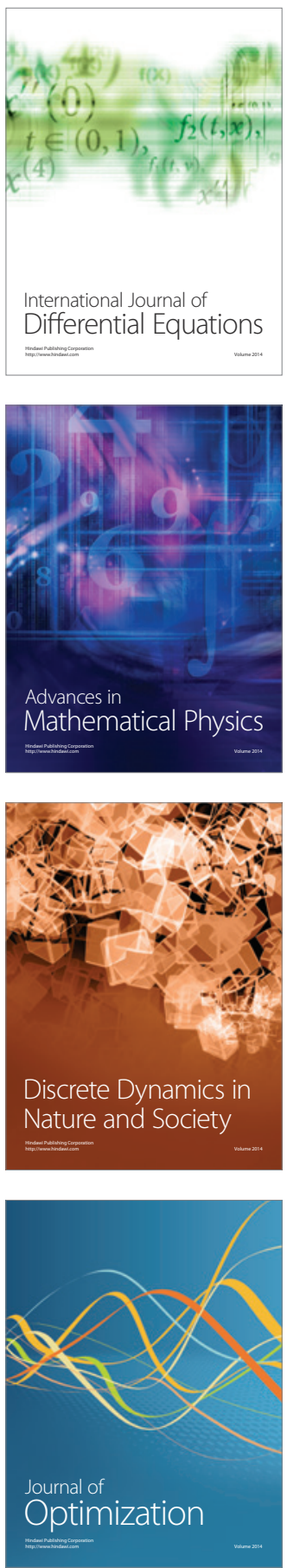